\title{
Estudio analítico-comparado sobre las políticas educativas en educación para la salud en la Unión Europea.
}

\author{
Laura Monsalve ${ }^{1 *}$, Jose Gallego ${ }^{2}$ y Jose Manuel Aguilar ${ }^{2}$ \\ ${ }^{I}$ Universitat de València $y^{2}$ Universidad de Almería
}

(Recibido el 2 julio 2013; Aceptado el 3 octubre 2013)

\begin{abstract}
RESUMEN: Este trabajo trata sobre un estudio comparado de las Políticas Educativas en material de Educación para la salud en los países de la Unión Europea con la finalidad de ver cómo se concretan en España. La Unión Europea en su legislación afirma que si se quiere avanzar como sociedad del conocimiento y competir eficazmente en una economía globalizada, para Europa es vital contar con una educación y formación de alta calidad. En la Unión Europea, la política educativa es competencia de cada país, pero entre todos fijan objetivos comunes y comparten las mejores prácticas. Por ello es motivo de este estudio comparar las Políticas educativas en materia de Educación para la salud en algunos países de la Unión Europea (España, Reino Unido, Francia y Alemania) y poder comparar las prácticas utilizadas en cada uno de ellos para poder de este modo coger lo mejor de cada país. El objetivo general que se persigue es analizar y comparar la situación de las políticas educativas en educación para la salud en las escuelas de la Unión Europea y en concreto las de Reino Unido, Francia, Alemania y España. A lo largo de este trabajo veremos que la educación para la salud en la escuela es un elemento de calidad así como un elemento necesario de educación integral en todas las etapas de la educación obligatoria.
\end{abstract}

Palabras clave: salud escolar, escuela promotora de salud, estudio comparativo, obligatoriedad, currículo escolar.

Analytical and comparative study on educational policies in health education in the European Union.

ABSTRACT: This paper deals with a comparative study of material Educational Policies in Health Education in the countries of the European Union for the purpose of seeing take shape in Spain. The EU legislation states that if you want to advance as a knowledge society and compete effectively in a global economy, Europe is vital to have an education and training of high quality. In the European Union, education policy is the responsibility of each country, but among all set common goals and share best practices. For this reason this study is to compare the educational policies on health education in some countries of the European Union (Spain, UK, France and Germany) and to compare the practices in each of them to this take the best of each country mode. The general objective pursued is to analyze and compare the situation of education policies in health education in the schools of the European Union and in particular the UK, France, Germany and Spain. Throughout this paper we will see that health education in school is 
a quality item as well as a necessary element of comprehensive education in all stages of compulsory education.

Keywords: school health, comparative study, compulsory, school curriculum, school health promoter.

\section{INTRODUCCIÓN}

Según la UE (Educación y formación: marco general de la UE, 2013) vemos que "la educación y la formación son elementos esenciales para la transformación de la Unión Europea (UE) en una de las sociedades y economías basadas en el conocimiento más competitivas del mundo". No se puede olvidar que la educación para la salud en la escuela cumple una labor indispensable para conseguir estos objetivos. Si se quiere avanzar como sociedad del conocimiento y competir eficazmente en una economía globalizada, para Europa es vital contar con una educación y formación de alta calidad (Educación y Formación 2020, 2009). En la UE, la política educativa es competencia de cada país, pero entre todos fijan objetivos comunes y comparten las mejores prácticas. Por ello es motivo de este estudio comparar las políticas educativas en materia de Educación para la salud en algunos países de la UE (España, Reino Unido, Francia y Alemania) y poder comparar las prácticas utilizadas en cada uno de ellos para poder de este modo coger lo mejor de cada país.

En las políticas de la Unión Europea (2013) la educación, la formación y la juventud desempeñan un papel fundamental en una economía basada en el conocimiento, ya que, al favorecer la aparición de una población altamente cualificada y adaptable, sostienen el crecimiento y el empleo. Además, refuerzan la cohesión social y la ciudadanía activa en la Unión Europea. A través de los programas en favor de la educación, la juventud y la salud, la Unión Europea desarrolla la dimensión europea, facilita la movilidad y fomenta la cooperación. Además, apoya y completa la acción de los Estados miembros, de conformidad con lo dispuesto en los artículos 165 y 166 del Tratado de Funcionamiento de la Unión Europea (Diario Oficial de la UE, 2010).

Siguiendo las directrices de la legislación de la UE (Unión Europea, 2013) en cuanto a educación y formación comprobamos que la promoción de la salud desde el ámbito público gubernamental implica derivar líneas de política pública y de investigación operativa para contribuir al cuerpo de conocimientos y su aplicación práctica con y para la población en general. Esto lo podemos ver desde la adopción de la Estrategia de Lisboa en 2000 (Consejo de Lisboa, 2000) que ha reforzado la cooperación política en materia de educación y formación, inicialmente a través del programa de trabajo "Educación y formación 2010" y después mediante "ET 2020", el marco estratégico de cooperación europea en educación y formación (Prats, 2010). Estas acciones de cooperación han propiciado la formulación de objetivos comunes y de iniciativas que abarquen todos los tipos de educación y formación así como todas las etapas de aprendizaje permanente (UE Educación y Formación, 2010). 
Cuentan con el apoyo de una serie de redes y agencias que realizan acciones en materia de educación y formación, como la Agencia Ejecutiva en el ámbito educativo, audiovisual y cultural y el Instituto Europeo de Innovación y Tecnología.

En 2009, el Consejo de Europa elaboró el marco estratégico para la cooperación europea en el ámbito de la educación y la formación bajo el lema "Europa 2020. Una estrategia para un crecimiento inteligente, sostenible e integrador" (Unión Europea Europa 2020, 2010)

\section{DESCRIPCIÓN}

Las Naciones Unidas han reconocido el potencial de las escuelas para influir no sólo en la situación educativa de la juventud, sino también en su salud y en su desarrollo económico (Estatutos de la Red SHE, 2007). Según este escrito esto se hace evidente en la importancia que se le ha dado a la educación primaria universal y en la eliminación de la disparidad de género tanto en la educación primaria como secundaria, dentro de los Objetivos de Desarrollo del Milenio (Diario Oficial de la Comunidades Europeas, 2012). Según la Red de Escuelas para la Salud en Europa existe relación entre la buena salud, los resultados educativos y la finalización de los estudios. También indican que estas relaciones implican que la mejora en la eficacia en un sector puede potencialmente beneficiar a otro sector, de forma que las escuelas sean un importante lugar tanto para la educación como para la salud. Por tanto existe una evidencia cada vez mayor de que los enfoques son más eficaces cuando incluyen la educación para la salud dentro de un modelo de escuela global. Estos crean un entorno de apoyo físico, social y de aprendizaje que involucra al alumnado, a las familias, a las comunidades y a las organizaciones. La enseñanza/aprendizaje en clase sobre cualquier tema de salud, como por ejemplo, la alimentación saludable o salud mental y emocional, requerirá la unión con iniciativas más amplias sobre la vida escolar en general y la comunidad, si éstas quieren tener un impacto sobre las conductas relacionadas con la salud o la situación de la misma a largo plazo (Red SHE, 2007).

La revisión de la literatura internacional (St Leger et al, 2001) apoya la eficacia del enfoque de las escuelas para la salud, en términos de los resultados que puede generar. Así mismo investigaron acerca de los efectos que tenía la escuela, como un todo, en los comportamientos de salud del alumnado.

Sus resultados también sirvieron para apoyar el enfoque de escuelas promotoras de salud. Stewart-Brown en 2006, concluyeron que los programas escolares que eran eficaces en cambiar la salud juvenil o los comportamientos relacionados con la salud fueron aquellos que implicaban actividades en más de un ámbito.

Tradicionalmente, la Educación para la Salud se ha trabajado desde siempre en la escuela por el profesorado de una manera un tanto incidental, ya que va estrechamente ligada al desarrollo personal y la autonomía de los alumnos (Talavera, 2008). Es por eso que, organismos internacionales como la OMS en colaboración con la UNESCO y la UNICEF decidieron, ya en el año 1978 en 
una reunión de expertos en Alma-Ata, adoptar algunas iniciativas con el fin de promocionar la educación para la salud en las escuelas, firmando la Declaración de Alma (Conferencia Internacional sobre Atención Primaria de salud, 1978).

Lo que posteriormente fue ratificado como la Carta de Ottawa (OMS, 1986), donde se reconocía la Educación para la salud como una de las herramientas para conseguir un estado completo de salud para todos.

Según la Red de Escuelas para la salud (2007), a nivel Europeo las administraciones educativas y sanitarias, conscientes de la importancia de la promoción y la educación para la salud en el medio escolar, abrieron un amplio cauce de actuación para el desarrollo de la Educación para la salud con las reformas llevadas a cabo en los últimos años.

En España por primera vez se aborda el tema en la Ley 14/1986, de 25 de Abril, General de Sanidad (Ley General de Sanidad) en la Ley Orgánica 1/1990, de 3 de octubre de 1990, de Ordenación General del Sistema Educativo (LOGSE) y recientemente continúa con el desarrollo de la Ley Orgánica 2/2006, de 3 de mayo, de Educación (LOE). Por otro lado, y de forma complementaria, la Ley General de Sanidad especifica la prioridad de la Promoción de la Salud y la Educación para la Salud dentro del Sistema Nacional de Salud, así como la colaboración estrecha del personal de Atención Primaria de Salud con los sectores de población en el que los Centros de salud están inmersos. Este aspecto ha quedado reforzado con la publicación del Real Decreto 1030/2006 de 15 de Septiembre, por el que se establece la cartera de servicios comunes del Sistema Nacional de Salud.

Por primera vez de forma explícita en el año 1990, se realizó el desarrollo legislativo de la Ley Orgánica General del Sistema Educativo (LOGSE), se estableció que la Promoción de la Educación para la Salud es un tema transversal que debe estar presente en todas las áreas curriculares.

En todas las etapas de la LOE, se abordan aspectos importantes para la Promoción de la Educación para la Salud en las áreas de: Conocimiento del Medio, Ciencias de la Naturaleza, Educación Física y Educación para la Ciudadanía. Vemos que en todos los niveles de la enseñanza obligatoria se establecen objetivos tendentes a desarrollar las capacidades afectivas y psicosociales del alumnado, a que se valoren y respeten los aspectos de género y la igualdad de derechos y oportunidades entre hombres y mujeres, a afianzar los hábitos de cuidado y salud corporales y a valorar críticamente los hábitos sociales relacionados con la salud, el consumo, el cuidado de los seres vivos y el medio ambiente. Todo esto lo pone de manifiesto la LOE ya que lo recoge entre sus fines y principios la transmisión de aquellos valores que favorezcan la libertad personal, la responsabilidad, el ejercicio de la ciudadanía democrática, la solidaridad, la tolerancia, la igualdad, el respeto y la justicia, que constituyen la base de la vida en común.

\section{MÉTODO}

Es evidente que el papel principal de las escuelas es la enseñanza/aprendi- 
zaje del contenido curricular. Pero la escuela también tiene un gran potencial en la promoción de la salud y el desarrollo de la infancia, de la juventud, de las familias y en general de toda la comunidad educativa. Una estrategia construida sobre el enfoque de escuelas promotoras de salud ayuda a: gestionar la salud y las cuestiones sociales; mejorar el aprendizaje del alumnado; mejorar la eficacia de la escuela.

Con este trabajo pretendemos hacer una revisión bibliográfica de las Políticas Educativas en Educación para la salud en los Países de España, Reino Unido, Francia y Alemania para posteriormente realizar un estudio analítico-comparado.

Para la consecución de los objetivos establecidos utilizaremos una metodología comparada con el fin de poder comparar cual es el estado de la cuestión establecido en torno a las Políticas Educativas en Educación para la Salud en Europa y España.

La metodología seguida en este estudio parte del método comparativo de carácter deductivo que, partiendo del modelo presentado por G.F Bereday y F.Hilker, incorporó luego aportaciones de García Garrido (1990).

Por lo que respecta a la delimitación temporal de la investigación, la recogida de información se ha extendido hasta agosto del 2012. Así pues, aparecen recogidas todas las normas, proyectos o actuaciones que están vigentes en dichas fechas. Todas las publicaciones que se realicen a partir de septiembre del 2012 no estarán recogidas en este trabajo.

\section{RESULTADOS}

A lo largo de este estudio hemos podido analizar en España, Reino Unido, Francia y Alemania la existencia de legislación en Educación para la salud. De estos datos cabe destacar que el país con más textos legales que hace referencia a la eps es España. En concreto en 13 documentos legales se alude a la eps. Todos ellos redactados desde 1978 hasta el 2012. En 1979 encontramos la Constitución, en 1985 la LODE, en 1986 la General de Sanidad, en 1989 el acuerdo entro los ministerios de Educación y Sanidad, en 1990 la LOGSE que introduce la eps como eje transversal, en el 2000 la transferencia en materia educativa no universitaria a las Comunidades Autónomas, en 2006 la LOE, en 2006 el Real Decreto de programas de eps, en 2006 los contenidos mínimos de infantil, primaria y secundaria y en 2012 los Reales decretos que modifican a los de primaria y secundaria. Seguidamente situamos a Francia con 12 textos entre 1975 y el 2011. En 1975,1978 y 1981 encontramos el Plan de estudios, en 1989 la Ley de Política Educativa Nacional, en el 2000 el protocolo nacional sobre la organización y emergencias de en las escuelas, en 2002 el acuerdo entre los ministerios de Educación y el Instituto nacional de prevención y educación para la salud, en 2003 la Ley de educación sexual en las escuelas, colegios y Liceos, en 2004 la Ley de Prevención y educación para la Salud, en 2005 el Plan de estudios, en 2010 el acuerdo de la Dirección de la Escuela de Educación y el INPES y en 2011 la Ley educativa dónde se establecen directrices nacionales de política 
educativa. En tercer lugar está Reino Unido con 8 textos legales. Aquí tenemos que destacar que el texto más antiguo que hace referencia a la eps, es de 1909, que establecería la obligatoriedad de la inclusión de la eps en la escuela del Reino Unido, éste es seguido en 1944 por Education Act, en 1988 la Ley de Reforma Educativa, en 1993 Education Act, en 1995 Education reform act, en 1997 la Ley de Educación, en 2006 la Ley de cuidado de niños-infantil y en 2001 se hace una revisión del currículo nacional para las escuelas primarias y secundarias. Por último situamos a Alemana con 6 textos legales, todos ellos comprendidos entre 1979 y el 2008. En 1979 se sitúa la Ley Fundamental de Educación, en 1990 la Ley del Bienestar de la infancia y la juventud, en 1992 se modifica la ley anterior, en 1996 se cran los Kindergarten, en 2004 la Ley de expansión de guarderías infantiles y por último en 2008 la Ley de promoción de la infancia. Cabe destacar que no existe relación entre mayor número de legislación con un mayor protagonismo de la eps en la escuela como veremos en los sucesivas tablas.

En cuanto a la existencia de una materia obligatoria de eps, en España en la etapa de educación infantil no existe materia propia de eps pero se trabaja en todo el currículum escolar de manera transversal. Pero la elección de unos temas transversales u otros va a depender de los valores que son de relevancia para el propio centro. Se trabajan los siguientes aspectos: hábitos higiénicos, alimentación, actividad física y tiempo libre, prevención de accidentes, salud mental, sexualidad, consumo y medios de comunicación y medio ambiente y salud. En educación primaria no tienen materia independiente pero se trabaja de manera transversal. Se puede trabajar en las siguientes materias: conocimiento del entorno natural, social y cultural; educación física y Educación para la ciudadanía. En Reino Unido en primaria la eps no es obligatoria pero está en el plan de estudios y es de obligado cumplimiento abordarla a través de las diferentes materias. En esta etapa no tiene asignatura independiente pero se trabaja de manera transversal en todas las demás. En secundaria sí que tiene materia independiente: ciudadanía, educación personal, social y salud y la otra materia es educación sexual. Además de tener estas dos materias independientes se trabajan transversalmente en las materias de ciencias y educación física. Es obligatorio cursarlas pero en el caso de la educación sexual los padres tienen la posibilidad de sacar a sus hijos de la clase. En Francia se trabaja de manera transversal. No tienen materia propia. Pero es la eps es obligatoria por lo tanto se trabaja desde diversas materias. En educación infantil el área para trabajar la eps en esta etapa es comunicarse con el cuerpo y descubrir el mundo. En la escuela primaria se trabaja la eps a través de las materias de educación cívica y educación sexual que en Francia es obligatoria pero integrada en otras materias. Es en el área de ciencias dónde se hace mayor hincapié. En educación secundaria la eps se enseña en biología y educación cívica. La educación sexual es obligatoria y los padres no pueden optar a sacar a sus hijos fuera. No es una materia independiente pero está cubierta por otras disciplinas. La eps es obligatoria en la escuela Francesa y se enseña integrada en otras materias. Alemania tiene un programa fuerte de eps, cuentan con el programa nacional Educación y Salud que pretende apoyar a la escuelas 
en la promoción y prevención de la salud. Todos los centros de educación infantil, primaria y secundaria participan de este programa. En educación infantil se trabaja transversalmente desde el área del desarrollo personal y social, el área del cuerpo, movimiento y salud y desde el área de la naturaleza. La educación sexual es obligatoria en Alemania durante la educación primaria y la eps se trabaja transversalmente desde biología, deporte, educación cívica, tratamiento de la naturaleza y del propio cuerpo. En secundaria se trabaja también de manera transversal en biología, educación física y deporte y educación cívica. La eps está dentro de la política educativa de este país, ya que tiene unos resultados positivos que elevan la calidad de la escuela. Cabe destacar que Alemania es el único país que los profesores coordinadores del programa reciben una reducción lectiva y financian personal externo para apoyar el proyecto.

Tabla 1. Contenidos que Trabaja la Eps en la Escuela. Elaboración Propia

\begin{tabular}{|c|c|}
\hline \multicolumn{2}{|c|}{ CONTENIDOS QUE TRABAJA LA EpS EN LA ESCUELA } \\
\hline ESPAÑA & $\begin{array}{l}\text { Actividad física y alimentación saludable } \\
\text { Bienestar y salud emocional .Salud mental en la infancia y adolescencia } \\
\text { Prevención de trastornos de la conducta alimentaria } \\
\text { Educación sobre drogodependencias: alcohol, tabaco y drogas ilegales } \\
\text { Hábitos higiénicos } \\
\text { Salud bucodental } \\
\text { Educación afectivo sexual. Prevención de embarazos no deseados } \\
\text { Seguridad y prevención de riesgos, lesiones y accidentes }\end{array}$ \\
\hline REINO UNIDO & $\begin{array}{l}\text { Promoción de la salud y la nutrición } \\
\text { Almuerzos escolares } \\
\text { Fomento de la actividad física } \\
\text { Educación de drogas y alcohol } \\
\text { Educación sexual y bienestar } \\
\text { La salud mental y emocional (incluyendo anti-bullying) } \\
\text { Alimentación y fitnes (incluyendo el desarrollo de una política relevante, el } \\
\text { consumo de alimentos y la orientación al fitnes, incluyendo la salud oral } \\
\text { El desarrollo de las relaciones personales } \\
\text { Uso de sustancias y mal uso (incluyendo el tabaco, el alcohol, las drogas legales e } \\
\text { ilegales } \\
\text { Medio ambiente (interno y externo) } \\
\text { Seguridad (incluyendo protección solar, seguridad e intereses) } \\
\text { Higiene, gérmenes y baño en la escuela }\end{array}$ \\
\hline
\end{tabular}




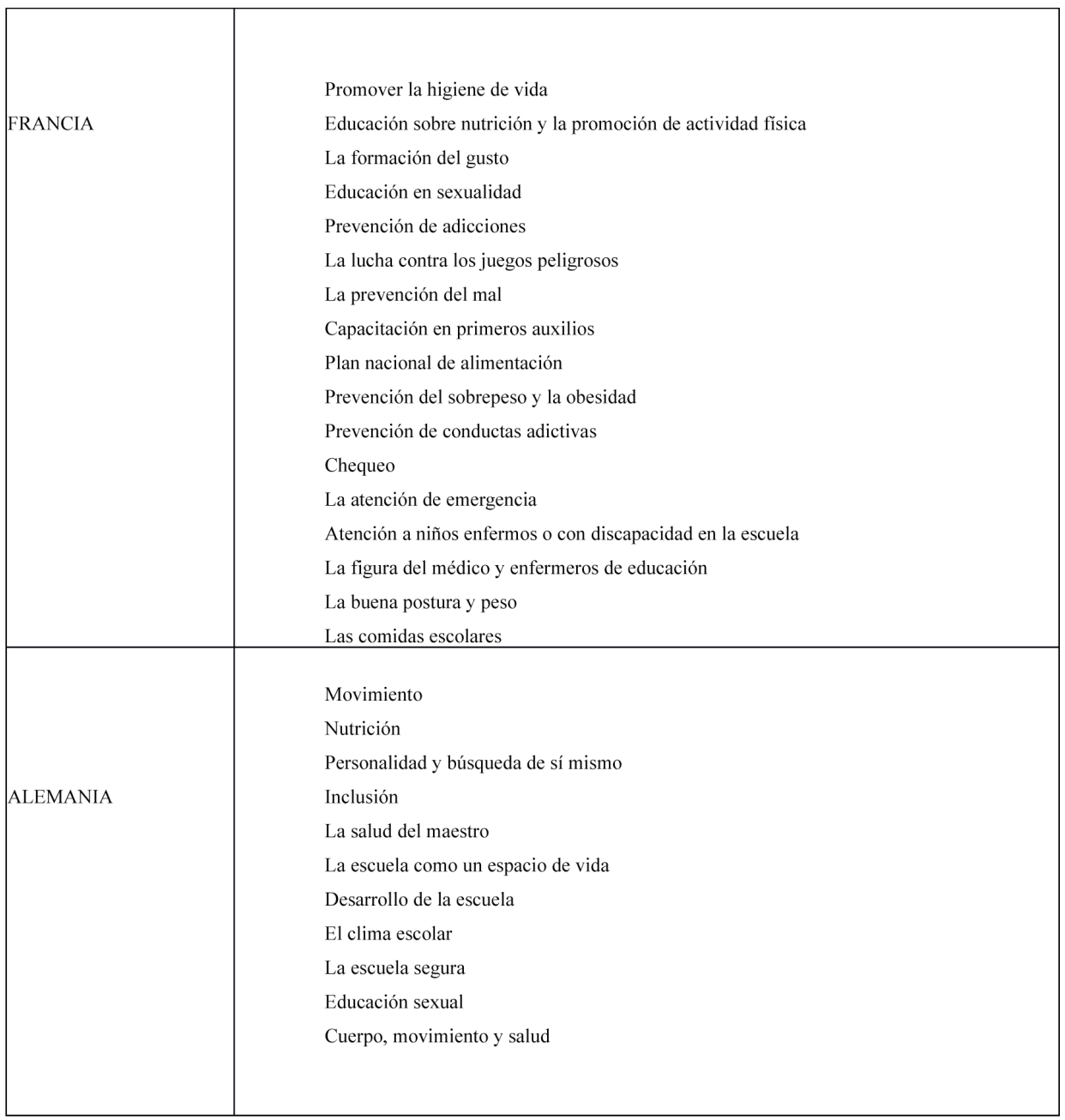

Por último, otro elemento analizado en este estudio han sido los contenidos de eps existentes en los currículos de los países estudiados.

De España podemos destacar que abarca todos los contenidos necesarios para abordar de una manera completa la eps. Pero en este modelo no se tiene en cuenta la salud del maestro ni el entorno. En Reino Unido ocurre lo mismo pero ellos sí que tratan el tema del medio ambiente. Francia es el país que más variedad de contenidos presenta. Cabe destacar la importancia que le dan a la formación del gusto, las conductas violentas y los juegos peligrosos. Son los únicos que capacitan en primeros auxilios, hacen chequeos en las escuelas, tienen enfermeros y médicos de educación en las escuelas y esto hace que este modelo sea un modelo a seguir. Alemania se centra en el movimiento, nutrición, personalidad, clima escolar, educación sexual y deja otros aspectos como las drogodependencias, uso 
de sustancias etc. Pero tiene a su favor que da mucha importancia al clima que se desarrolla en la escuela y contempla a todos los miembros de la comunidad educativa además que da importancia a la salud del maestro.

\section{DISCUSIÓN}

La escuela tiene la responsabilidad de integrar la promoción de la Educación para la Salud en el currículo escolar y esto significa sobre todo proporcionar el valor de la salud entre todos los grupos que integran la población y la comunidad escolar. Para ello debe de haber una política y legislación educativa en materia de educación para salud que desarrolle una normativa que lo apoye.

La promoción de la Educación para la Salud en la escuela es un elemento de calidad así como un elemento necesario de educación integral en todas las etapas de la educación obligatoria.

Actualmente en las escuelas los proyectos que se ponen en marcha para conseguir estos objetivos de salud tanto realizados por docentes como por profesionales externos son los siguientes: Mejora de los hábitos higiénicos, actividades de ocio y tiempo libre, salud bucodental, prevención de alteraciones de la columna vertebral, prevención de alteraciones de la visión, práctica deportiva, prevención y control del tabaquismo, prevención y control del tabaquismo, prevención de drogodependencias, educación afectivo-sexual, alimentación y hábitos de vida saludables.

Tras realizar un análisis sobre la Red de Escuelas para la Salud en los países que nos ocupa, llegamos a la conclusión que a pesar de que los cuatro países pertenecen a la Red SHE, en España y Reino Unido podemos encontrar una mayor información sobre las actividades que realizan a nivel nacional. Estos datos se pueden observar tanto en la página web de la Red SHE europea como en los ministerios de educación de los respectivos países. Sobre la Red Nacional de Escuelas para la Salud de Francia y Alemania en la página web de la Red SHE europea solamente indican el contacto nacional así como en la búsqueda en los ministerios de sanidad y educación de los respectivos países la información acerca de la red es escasa. A pesar que estos dos países tienen una fuerte política educativa en educación para la salud, incluso obligatoriedad en el currículo escolar de algunos ámbitos de la Educación para la salud.

Hemos podido comprobar a lo largo de este estudio que la Red de Escuelas para la Salud en Europa facilita el intercambio de información y buenas prácticas y favorece los contactos entre los países miembros. La colaboración internacional contribuye a minimizar la duplicidad de esfuerzos, y proporciona un marco que fomenta y propugna la innovación.

Los profesionales que trabajan en diversos sectores: sanitario, educativo, atención a la juventud, y otros sectores relacionados, pueden encontrar en la red de Escuelas para la Salud en Europa un apoyo importante para la puesta en marcha de proyectos y acciones escolares. Comprobamos que esto es cierto y que no se queda sólo en la teoría ya que los objetivos y contenidos a trabajar de 
la Educación para la salud en las escuelas de los países estudiados son comunes.

Existe una evidencia cada vez mayor de que los enfoques en la escuela son más eficaces cuando incluyen la educación para la salud dentro de un enfoque global de la escuela. Estos enfoques crean un entorno de apoyo físico, social y de aprendizaje, que involucra al alumnado, a las familias, a las comunidades y a las organizaciones. La enseñanza/aprendizaje en clase sobre cualquier tema de salud, como por ejemplo, la alimentación saludable o salud mental y emocional, requerirá la unión con iniciativas más amplias sobre la vida escolar en general y la comunidad, si éstas quieren tener un impacto sobre las conductas relacionadas con la salud o la situación de la misma a largo plazo.

\section{REFERENCIAS}

Consejería de salud (1989). Propuesta de Educación para la Salud en los Centros Docentes. Sevilla: Junta de Andalucía.

Declaración de Yakarta sobre la promoción de la salud en el siglo XXI (1997). Cuarta Conferencia Internacional sobre la Promoción de la Salud, Julio 2125. Yakarta: OMS.

Diario oficial de las comunidades europeas (2012). Programa de trabajo detallado para el seguimiento de los objetivos concretos de los sistemas de educación y formación en Europa. Bruselas: Unión Europea.

Diario oficial de la Unión Europea (2012). Informe del marco estratégico para la cooperación europea en el ámbito de la educación y la formación (ET 2020). Bruselas: Unión Europea.

El Consejo de Europa (1997). La eficacia de la enseñanza del inglés en la Unión Europea. Informe de un coloquio. París: Consejo de Europa.

Eurydice Unidad Alemana (1998). El sistema de educación en Alemania. Bruselas: Eurydice.

Eurydice (1995). Las estructuras de la educación y los sistemas de formación inicial en la Unión Europea. Luxemburgo: Oficina de publicaciones oficiales de las comunidades europeas.

Eurydice (2008). El sistema de educación en España. Luxemburgo: Oficina de publicaciones oficiales de las comunidades europeas. Consultado el 14-062012 en http://www.eurydice.org/.

Eurydice Europeo (2001). Los sistemas educativos en Europa: Francia. Luxemburgo: Oficina de publicaciones oficiales de las comunidades europeas. Consultado el 16-06-2012 en http://www.eurydice.org/.

Eurydice Francia (2008). El sistema de educación en Francia. Luxemburgo: Oficina de publicaciones oficiales de las comunidades europeas. Consultado el 18-06-2012 en http://www.eurydice.org/.

Eurydice Francia (2008). Dossiers d ' information sur les Systèmes d'Éducation dans les États Membres de la Communauté Européenne. Luxemburgo: Oficina de publicaciones oficiales de las comunidades europeas. Consultado el 18-06-2012 en http://www.eurydice.org/. 
Eurydice Francia (1996). Respuesta a la pregunta de red. Luxemburgo: Oficina de publicaciones oficiales de las comunidades europeas.

Eurydice Gran Bretaña (2010). Currículo y evaluación. Luxemburgo: Oficina de publicaciones oficiales de las comunidades europeas. Consultado el 22-062012 en http://www.eurydice.org/.

Eurostat (1990). Europa en cifras. Luxemburgo: Oficina de Publicaciones de las Comunidades Europeas, pp.3.

Eurostat (2001). Los niveles de educación en la UE. Luxemburgo: Oficina de Publicaciones de las Comunidades Europeas.

Enric Prats (2010). Equity and efficiency in the Lisbon Strateggy: a structural challenge in basic education systems in Europe. Revista Española de Educación Comparada, 16, 131-158

García Garrido, JL. (1990). Fundamentos de Educación Comparada. Madrid: Dykinson.

Ministerio de Educación, Cultura y Deporte (1998). Promoción de la salud. Glosario. Madrid: MECD.

Ministerio de Educación y Ciencia (2007c). La LOE entra en las aulas. Madrid: MEC. Consultado el 10/10/2012 en http://www.mec.es/mecd/gabipren.

Ministerio de Educación, Política social y Deporte y Ministerio de Sanidad y Consumo (2008). Guía para las administraciones educativas y sanitarias, criterios de calidad para el desarrollo de proyectos y actuaciones de promoción y Educación para la Salud en el Sistema Educativo. Madrid: Ministerio de Sanidad y Consumo.

Ministerio de Sanidad y Consumo (1999). Glosario de promoción de la salud. Madrid: Ministerio de Sanidad y Consumo.

Ministerio de Sanidad y Consumo (1999). Salud 21. Salud para todos en el siglo XXI. Madrid: Ministerio de Sanidad y Consumo.

Ministerio de Sanidad y Consumo (2000). Unión Internacional de Promoción de la Salud y Educación para la Salud. La evidencia de la eficacia de la Promoción de la Salud. Madrid: OMS.

Ministerio de Sanidad y Consumo (2006). Los determinantes sociales de la salud. Los hechos probados. Madrid: OMS.

Ministerio de Sanidad, Consumo; Ministerio de Educación y Ciencia (2006). Actividad fisica y salud en la infancia y la adolescencia. Madrid: Ministerio de Sanidad y Consumo.

Ministerio de Sanidad y Consumo (2007). Desarrollo, adolescentes y salud. Madrid: Ministerio de Sanidad y Consumo.

Ministerio de Sanidad y Consumo, Ministerio de educación y Ciencia (2008). Informe sobre criterios de calidad para el desarrollo de programas y actuaciones de Promoción y Educación para la Salud en el sistema educativo: una guía de actuación para centros educativos. Madrid: Ministerio de Sanidad y Consumo.

Ministerium Für Schul (2003) Jugend und Kinder des Landes Nordrhein-Westfalen: Bildungsvereinbarung. Düsseldorf: NRW. 
Organización Mundial de la Salud (1986). Carta de Ottawa para la promoción de la salud. Ginebra: OMS.

Organización Mundial de la Salud (1990). Conferencia sobre la promoción de la educación para la salud. 20-22 de septiembre. Estrasburgo: OMS.

Organización Mundial de la Salud (1990). Conferencia Europea de actuación para la salud. Dublín: OMS.

Organización Mundial de la Salud (1990). Conferencia sobre la promoción de la educación para la salud. 20-22 de septiembre. Estrasburgo: OMS.

Scottish Consultative Council on the Curriculum (1999). Curriculum design for the secondary stages. Dundee: Learning and Teaching Scotland.

Stewart-Browns (2006). What is the effectiveness of the health promoting schools approach? Copenhagen:WHO.

St Leger, L. (2001) Schools, health literancy and public health. Health Promotion International, 16, 197-205.

Talavera, M. (2008). La educación para la salud en la Red de Escuelas Promotoras de salud de la Comunidad Valenciana. Tesis doctoral. Universitat de València, Comunidad Valenciana, España. Consultada el 09/08/2012.

Europa 2020 (2010). Una estrategia para un crecimiento inteligente, sostenible e integrador. Bruselas: Unión Europea.

WHO (1986). Ottawa Charter for Health Promotion. Geneva, World Health Organization. Consultado el 15/02/2012 en: http://www.who.int/healthpromotion/conferences/previous/ ottawa/en 